\title{
Transient energy growth of channel flow with cross-flow
}

\author{
J. BENYZA , M.LAMINE , A. HIFDI \\ Laboratory of Mechanics, Faculty of Sciences Ain-Chock, University Hassan II-Casablanca, Morocco. \\ Email adresse : jawadbenyza@gmail.com
}

\begin{abstract}
The effect of a uniform cross flow (injection/ suction) on the transient energy growth of a plane Poiseuille flow is investigated. Non-modal linear stability analysis is carried out to determine the two-dimensional optimal perturbations for maximum growth. The linearized Navier-Stockes equations are reduced to a modified Orr Sommerfeld equation that is solved numerically using a Chebychev collocation spectral method. Our study is focused on the response to external excitations and initial conditions by examining the energy growth function $G(t)$ and the pseudo-spectrum. Results show that, the transient energy of the optimal perturbation grows rapidly at short times and decline slowly at long times when the crossflow rate is low or strong. In addition, the maximum energy growth is very pronounced in low injection rate than that of the strong one. For the intermediate crossflow rate, the transient energy growth of the perturbation, is only possible at the long times with a very high-energy gain. Analysis of the pseudo-spectrum show that the nonnormal character of the modified Orr-Sommerfeld operator tends to a high sensitivity of pseudo-spectra structures.
\end{abstract}

Keywords: Cross flow; Non-modal linear stability;

Poiseuille flow; Spectral method; Transient energy growth.

\section{Introduction}

The eigenvalue analysis is able to predict instability behavior for some fluid systems, such as RayleighBénard convection and Taylor-Couette flow [1]. Several theoretical papers show, for all Reynolds numbers the Couette and Poiseuille flows are unconditionally stable [2]. However, this approach does not correspond to experimental results for other problem $[3,4]$, in which the transition to turbulence is observed at $350<R e<370$ for Couette flow [3]. The gap between the eigenvalue analysis and experiments leads to the emergence of a new theory called: theory of non-modal stability [6]. This, we motivate to reproduce the results of Fransson and Alfredsson [5] by using the non-modal approach, in which, we focus on the response to initial conditions by examining the pseudo-spectra structures and the transient energy growths.

Fransson and Alfredsson [5] carried out a linear modal stability analysis of the plane Poiseuille flow with cross- flow. The authors made corrections to the problems discussed in $[3,4]$ and they proved that the stability of this problem depends on the choice of the velocity scale. In addition, they showed the stabilizing and the destabilizing effect of a uniform cross flow.

\section{Physical problem and Mathematical formulation}

\subsection{Physical problem}

Consider a plane channel flow of an incompressible fluid with the density, $\rho$, and the dynamic viscosity, $\mu$.The channel is formed by two porous parallel plates separated by a fixed distance $2 \mathrm{~d}$. The upper and lower plates are located, respectively, at $y^{*}=+h$ and $y^{*}=-h$. A uniform cross-flow (injection/suction) of constant velocity, $V_{0}$, is imposed on the channel walls in the transverse direction, $\mathrm{y}^{*}$. The injection at upper plate and suction at lower plate as shown in Fig.1.

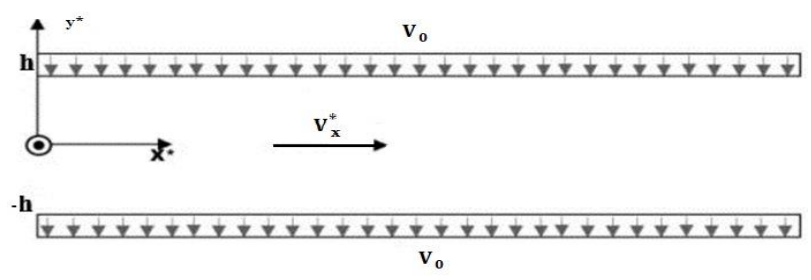

Figure 1 : Sketch of the flow configuration.

The governing equations that describe the flow of an incompressible Newtonian fluid are the Navier-Stokes and continuity equations. In non-dimensional form, they are written as:

- Continuity equation:

$$
\nabla \cdot \mathrm{V}^{*}=0
$$

- Navier-Stokes equations:

$$
\rho\left[\frac{\partial V^{*}}{\partial t}+\left(V^{*} . \nabla\right) V^{*}\right]=-\nabla P^{*}+\Delta V^{*}
$$

Boundary conditions:

$$
V_{x}^{*}\left(x^{*} ; \pm h ; t^{*}\right)=0 \quad ; \quad V_{y}^{*}\left(x^{*} ; \pm h ; t^{*}\right)=V_{0}
$$

where $\mathrm{V}^{*}, \mathrm{P}^{*}$, respectively, the velocity and the pressure.

Using reference variables $h, \rho U_{0}^{2}, U_{0}, \frac{U_{0}}{h}$ for, respectively, length, pressure, velocity and time, ( $U_{0}$ represents the maximum streamwise velocity), 
as follows non-dimensional variables:

$x=\frac{x^{*}}{h}, y=\frac{y^{*}}{h}, p=\frac{p^{*}}{U_{0}^{2}}, V_{x}=\frac{V_{x}^{*}}{U_{0}}, V_{y}=\frac{V_{y}^{*}}{U_{0}}, \mathrm{t}=\frac{U_{0} t^{*}}{h}$

The basic velocity in non-dimensional form can be written as:

$$
V_{x b}(y)=R_{c} \frac{y+\sinh ^{-1}\left(R_{c}\right) \exp \left(-R_{c} y\right)-\operatorname{coth}\left(R_{c}\right)}{1+\log \left[R_{c}^{-1} \sinh \left(R_{c}\right)-\mathrm{R}_{c} \operatorname{coth}\left(R_{c}\right)\right]}
$$

where $R_{c}=\frac{\rho V_{0} h}{\mu}$ is the cross-flow Reynold number related to $V_{0}$.

\subsection{Linear stability analysis}

For the perturbed flow, the velocity and pressure fields are expressed as the sum of a steady and a perturbation field, i.e.

$$
\left(\mathrm{V}_{x} ; V_{y} ; P\right)=\left(V_{x b}+u^{\prime} ; v^{\prime} ; P_{b}+p^{\prime}\right)
$$

The disturbance quantities $u^{\prime}, v^{\prime}$ and p' are assumed periodic and of the form:

$$
v^{\prime}(x, y, t)=v(y, t) e^{i \alpha x}
$$

The modified Orr-Sommerfeld equation with their boundary conditions are:

$$
\begin{gathered}
\left(D^{2}-\alpha^{2}\right) \frac{\partial v}{\partial t}=\left[i \alpha D V_{x b}+\frac{1}{\mathrm{R}_{e}}\left(D^{2}-\alpha^{2}\right)^{2}+\left(\frac{R_{c}}{R_{e}} D-i \alpha V_{x b}\right)\right] v \\
v( \pm 1, t)=D v( \pm 1, t)=0
\end{gathered}
$$

Where $R_{e}=\frac{\rho U_{0} h}{\mu}$ is the Reynolds number related to $U_{0}$.

For modal approach consists a reducing the initial values problem to that of the eigenvalues as follows:

$$
v(y, t)=q(y) e^{-\omega t}
$$

Then the problem (7a) (7b) with the initial values can be written as

$$
\omega B q=A q
$$

where $A$ and $B$ are matrix operators that depend on $\alpha$, $R_{e}$ and $R_{c}$.

$A=\left[i \alpha D V_{x b}+\frac{1}{\mathrm{R}_{e}}\left(D^{2}-\alpha^{2}\right)^{2}+\left(\frac{R_{c}}{R_{e}} D-i \alpha V_{x b}\right)\left(D^{2}-\alpha^{2}\right)\right]$

$B=\left(\alpha^{2}-D^{2}\right)$

For non-modal approach, stability is redefined in a broader sense as the response to general input variables, including initial conditions, impulsive and continuous external excitations, the pseudospectra can also be defined in other equivalent ways [7].

$$
\sigma_{\varepsilon}(L)=\left\{z \in \mathbb{C},\left\|(z I-L)^{-1}\right\| \geq \frac{1}{\varepsilon}\right\}
$$

where $L=B^{-1} . A$ is the operator non-normal and $\sigma_{\varepsilon}(L)$ is the $\varepsilon$-pseudospectrum

Finally, we obtain the linear initial value problem:

$$
\frac{d q(t)}{d t}=L q(t)
$$

Its energy growth of the perturbation at time $t$ is measured by the ratio $g(t)$ between the energy norm $\|q(t)\|_{E}^{2}$ of the perturbation at time $t$ and its initial norm $\|q(0)\|_{E}^{2}:$

$$
g(t)=\frac{\|q(t)\|_{E}^{2}}{\|q(0)\|_{E}^{2}}
$$

For a given set of values of $\alpha, \mathrm{Rc}$, and Re, the maximum possible energy amplification at time $t$ over all possible initial combinations of the $\mathrm{L}$ eigenfunctions, is denoted by

$$
G(t)=\max _{q(0) \neq 0}[g(t)]
$$

The maximum growth for all time $\mathrm{t}$ is denoted by

$$
G^{\max }=\sup _{t \geq 0} G(t)
$$

\section{Numerical method}

The eigenvalue problem, (10) is solved numerically using the spectral collocation method based on Chebychev polynomials evaluated in N Gauss-Lobatto collocation points [6-8].

\section{Results}

Figure. 2 presents the effect of the cross-flow Reynolds number on the pseudospectral boundaries and also the spectra in the $\omega$ for $\alpha=1.0$ and $\operatorname{Re}=6000$. It is observed that the number of eigenvalues on each branch depends on the cross-flow Reynolds number. In addition, the sensibility of the vicinity of the intersection is dependent on the cross-flow Reynolds number.

In figure. 3 exhibits the evolution of the energy growth function $G(t)$ as a function the time for different values of $\operatorname{Rc}$ at $\alpha=1.0$ and $\operatorname{Re}=6000$. It can be seen that; the transient energy of the optimal perturbation grows rapidly at short times when the cross-flow rate is low or strong and decline slowly at long times when the crossflow is intermediate.

Figure. 4 represents wave numbers as a function of $\mathrm{Re}$ for several values of $\mathrm{Rc}=0.1 ; 100 ; 600$. It can be observed, in contrast to the important cross-flow Reynolds RC, the transient growth of wavelength disturbance became more pronounced in that in the small Rc. Also, as Rc incresed the flowins move towards the dangerous mode corresponding to $\alpha=1$. Figure. 5 depicts the maximum growth in the (Rc, Re) plane for $\alpha=1$. 
Results show that the region of high maximum energy growth expanded with $(\mathrm{Rc}, \mathrm{Re})$. this result in also given in [5] using the modal stability.
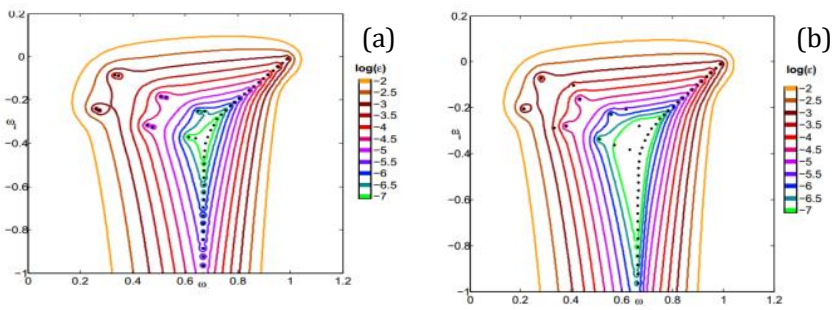

Figure 2 : Pseudospectra with $\alpha=1, \operatorname{Re}=6000$ (a) $R c=0,1,(b) R c=1$ and $(c)$ $R c=10$ The outermost and innermost contours represent levels from $\varepsilon=10^{-2}$ to $10^{-7}$
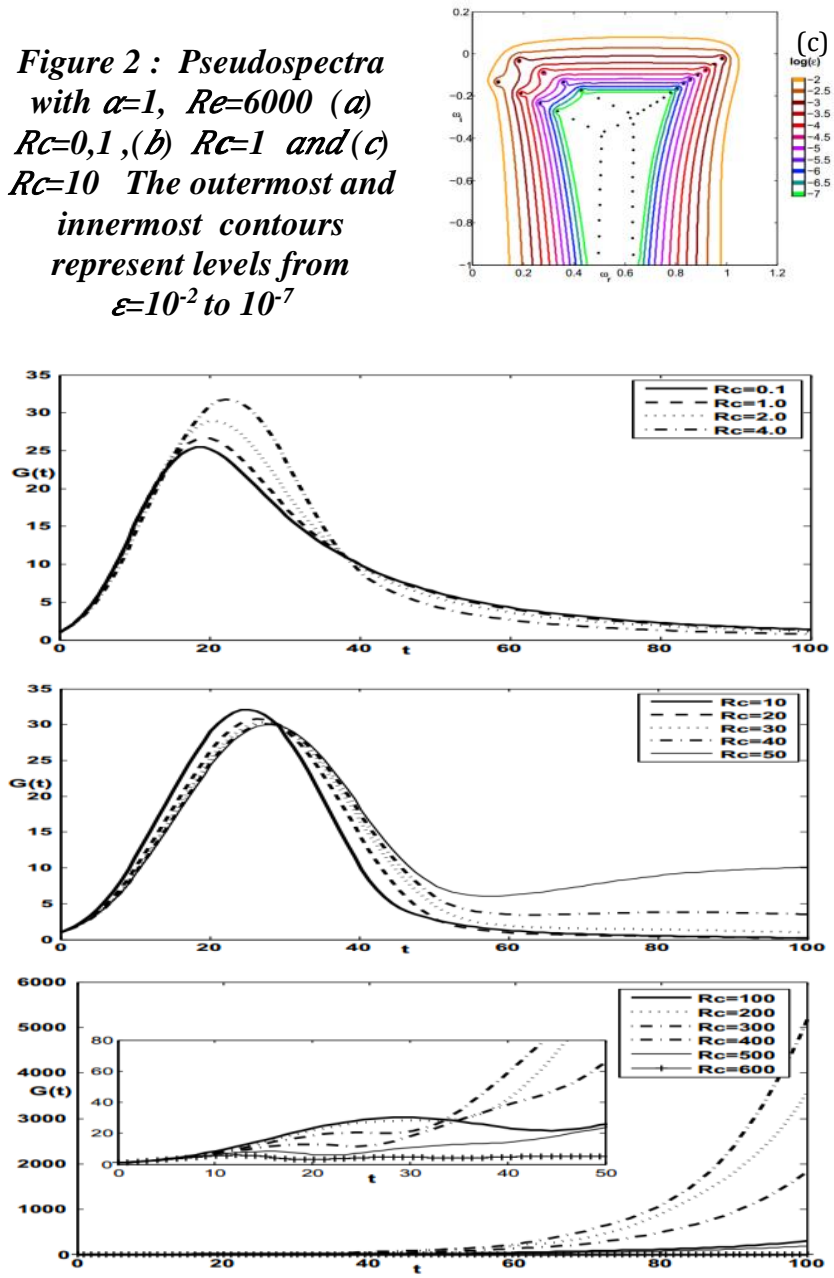

Figure 3 : growth $\mathbf{G}(\mathbf{t})$ versus time with $\alpha=1$, $R e=6000$ various $\mathrm{Rc}$.
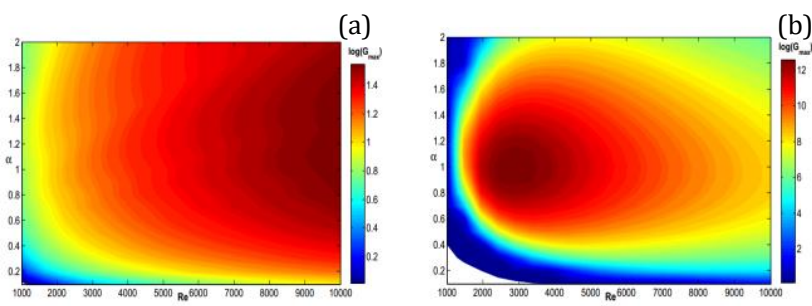

Figure 4 : Contour plots of the maximum gain $\log \left(G \_\max \right)$ in the $(\alpha, R e)$ with (a) $R c=0,1$, (b) $\mathrm{R} c=50,(c) R c=100$ and (d) $\mathrm{Rc}=600$

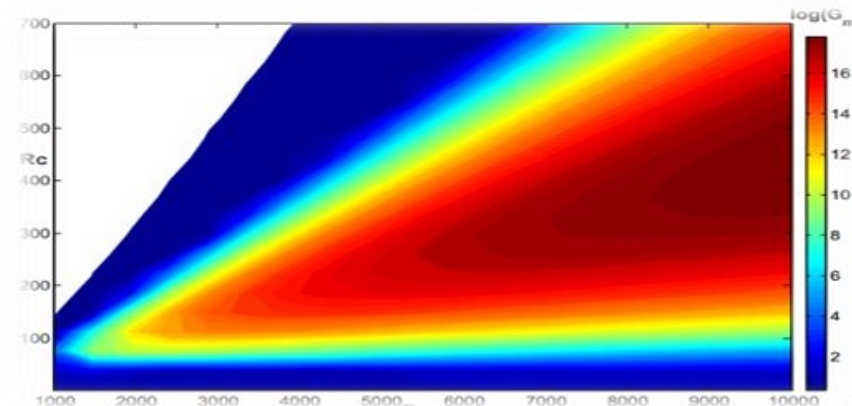

Figure 5 : Contour plots of the maximum gain $\log \left(G \_\max \right)$ in the $(\mathrm{Rc}, R e)$-plane

\section{Conclusion}

The present work has considered the effect of a uniform cross flow (injection/ suction) on the transient energy growth of a plane Poiseuille flow is investigated. Nonmodal linear stability analysis is carried out to determine the two-dimensional optimal perturbations for maximum growth. Results show that, the transient energy of the optimal perturbation grows rapidly at short times and decline slowly at long times when the cross-flow rate is low or strong. In addition, the maximum energy growth is very pronounced in low injection rate than that of the strong one. For the intermediate cross-flow rate, the transient energy growth of the perturbation, is only possible at the long times with a very high-energy gain. Analysis of the pseudo-spectrum show that the nonnormal character of the modified Orr-Sommerfeld operator tends to a high sensitivity of pseudo-spectra structures.

\section{References}

[1] TAYLOR, G. I. 1923 Stability of a viscous liquid contained between two rotating cylinders. Phil. Trans. R. Soc. Lond. A 223, 284-343.

[2] P. G. Drazin and W. H. Reid (1981) "Hydrodynamics Stability, First edition". Cambridge University Press.

[3] Tillmark, N. \& Alfredsson, P. H.1992 Experiments on transition in plane Couette flow. J. Fluid Mech. 235, 89-102.

[4] D. Max Sheppard. Hydrodynamic stability of the flow between parallel porous walls.Phys. Fluids, 1972.

[5] J. H. M. Fransson and P. Henrik Alfredsson. On the hydrodynamics stability of channel flow with cross flow. Physics of Fluids, 15(2), pp. 436-441.

[6] P.J. Schmid, Nonmodal stability theory, Annu. Rev. Fluid Mech. 39 (2007) 129-162.

[7] L.N. Trefethen, M. Embree, Spectra and Pseudospectra: The Behavior of Nonnormal Matrices and Operators, Princeton University Press, 2005.

[8] RAFIKI,A ; HIFDI,A et OUAZZANI,T ,M, Stabilité hydrodynamique linéaire des écoulements plans de Poiseuille de fluides viscoélastiques en présence d'un champ magnétique transversal, CFM2013, 21ème Congrès Français de Mécanique, Bordeaux, Français, 2013-12-20. 